\title{
ANALYSIS OF WATER HAMMER ATTENUATION IN THE BRUNONE MODEL OF UNSTEADY FRICTION
}

\author{
BY \\ E. YAO (Department of Engineering Mathematics, Dalhousie University, 1340 Barrington St., \\ Halifax, NS, Canada, B3J 1B7),
}

G. KEMBER (Department of Engineering Mathematics, Dalhousie University, 1340 Barrington St., Halifax, NS, Canada, B3J 1B7),

AND

D. HANSEN (Department of Civil and Resource Engineering, Dalhousie University, 1360 Barrington St., Halifax, NS, Canada, B3H 4R2)

\begin{abstract}
A multiple-scales asymptotic analysis is used to describe the attenuation of a water hammer pressure wave in the Brunone model of unsteady friction. The method is applied to water hammer caused by sudden valve closure in water reservoir pipelines. The analytical results explain the parametric dependence of the Brunone unsteady friction pressure-wave attenuation. It is also found that viscous head in an extended steady friction model may provide an alternative to the unsteady friction basis for increased attenuation in cases where the attenuation has a weak spatial dependence and is primarily time-dependent. All results are numerically verified using the method of characteristics.
\end{abstract}

1. Introduction. The development of the Brunone model of unsteady friction began from experimental studies ([5, [11, 9]) with the intention of understanding earlier experimental results on oscillatory unsteady pipe flows. These studies were followed by a discussion paper [6] where these results were summarized. The overall thrust of the discussion in [6] was twofold: (i) Quasi-steady friction with the Reynolds number computed as the flow velocity evolves was insufficient to describe pressure-wave attenuation and other water hammer features. (ii) They empirically resolved this insufficiency by including an unsteady friction proportional to local flow acceleration during the phase of the flow when the kinetic energy is increasing. This unsteady friction model has a natural jump discontinuity between the forward and backward flow phases, and this was later smoothed through a physical argument [7].

Received June 18, 2012.

2010 Mathematics Subject Classification. Primary 74G10, 93C70.

E-mail address: Edward.Yao@dal.ca

E-mail address: Guy.Kember@dal.ca

E-mail address: David.Hansen@dal.ca 
The objective of this paper is to develop an analytical pressure-wave attenuation formula for the Brunone model of unsteady friction presented in [7] and studied elsewhere, for example, in 12, 11 and 4. The results found here explain the physical nature of the increased pressure-wave attenuation according to the Brunone unsteady friction model in comparison with the standard steady friction model.

The water hammer is assumed to be initiated by a sudden valve closure. The steady friction component of the model considered here is an extended steady friction model where the non-linear Darcy-Weisbach friction is generalized to include a linear viscous friction term. A practically useful result predicted by the form of the analytical pressurewave attenuation and numerically verified is that the extended friction model may be used to describe the pressure-wave attenuation.

2. Water hammer equations. The unsteady momentum equation [7] based on experiments detailed in [6] and further modified to a form suitable for a wider range of applications [12] (using subscripts for partial derivatives) is

$$
g h_{x}+v v_{x}+v_{t^{\prime}}+\frac{g}{L}(r v+s v|v|)+\frac{k}{2}\left(v_{t^{\prime}}+c_{p} \operatorname{sign}(v)\left|v_{x}\right|\right)=0 .
$$

The head is $h(x, t)$, fluid velocity is $v(x, t), L$ is pipe length and $r$ and $s$ are constant steady friction parameters proportional to the pipe length $L$ [10]. The extended steady friction term $r v+s v|v|$ in (11) includes the linear term $r v$ [10] which extends the steady friction model to partially developed turbulent and laminar flow regimes. The steady friction model $r v+s v|v|$ is hereafter referred to as the 'extended' steady friction model. The unsteady friction model [6] includes the term proportional to $v_{t^{\prime}}+c_{p} \operatorname{sign}(v)\left|v_{x}\right|$ where $k$ is the Brunone friction coefficient and $c_{p}$ is the wave celerity detailed below.

The continuity equation (cf. [10]) is

$$
\frac{c_{p}^{2}}{g} v_{x}+v h_{x}+h_{t^{\prime}}+v \sin (\theta)=0
$$

with wave celerity $c_{p}=\sqrt{K / \rho}, K=E_{v} /\left(1+D_{c} E_{v} /(w E)\right.$, water bulk modulus $E_{v}$, pipe material modulus $E$, pipe wall thickness $w$, and pipe slope $\theta$.

The water hammer equations are considered here for water hammer initiated by a valve closure and applied to the physical model of valve-closure water hammer described in [1].

3. Water hammer application. An initial head $h=h_{2}+(1-x / L)\left(h_{1}-h_{2}\right)$ initially maintains a steady-state flow velocity $v=v_{\infty}$. After $t=0$, the velocity at the pipe downstream boundary $x=L$ is zeroed by suddenly closing a valve, and this initiates a water hammer wave at $x=L$ that travels backward toward the upstream end $x=0$. The boundary and initial conditions are

$$
\begin{aligned}
h(x, t=0)=h_{2}+(1-x / L)\left(h_{1}-h_{2}\right), v(x, t=0) & =v_{\infty} \\
h(x=0, t>0)=h_{1}, v(x=L, t>0) & =0 .
\end{aligned}
$$

At steady-state, the velocity is zero and the head is constant at $h_{1}$. 
Water hammer initiated by valve closure was studied in [14] in the case of steady friction and is generalized here to include the Brunone model of unsteady friction. A physical application presented in [1] is considered after reduction to dimensionless form in the following section is completed.

4. Nondimensionalization. The dimensional head and velocity of equations (11) and (2) are rescaled to vary between zero and unity via $H=\left(h-h_{2}\right) / h_{12}$ and $V=v / v_{\infty}$ where $h_{12}=h_{1}-h_{2}$ is applied head. The pipe length $L$ is rescaled to unity with $X=x / L$ and $0 \leq X \leq 1$. An inertial time-scale $v_{\infty} / g$ is scaled by the hydraulic gradient $h_{12} / L$ yielding the dimensionless time-scale $T=L v_{\infty} /\left(g h_{12}\right)$, and defining $\tau=t^{\prime} / T$ yields the dimensionless momentum and continuity equations

$$
\begin{array}{r}
H_{X}+C_{1} V V_{X}+V_{\tau}+C_{2} V+C_{3} V|V|+B_{1} V_{\tau}+B_{1} B_{2} \operatorname{sign}(V)\left|V_{X}\right|=0 \\
V_{X}+C_{1} C_{4} V H_{X}+C_{4} H_{\tau}+C_{5} V=0
\end{array}
$$

and dimensionless parameters

$$
\begin{array}{r}
C_{1}=\frac{v_{\infty}^{2}}{g h_{12}}, C_{2}=\frac{r v_{\infty}}{h_{12}}, C_{3}=\frac{s v_{\infty}^{2}}{h_{12}}, B_{1}=\frac{k}{2}, B_{2}=\frac{c_{p} v_{\infty}}{g h_{12}} \\
C_{4}=\left(\frac{g h_{12}}{c_{p} v_{\infty}}\right)^{2}, C_{5}=\frac{g L \sin \theta}{c_{p}^{2}} .
\end{array}
$$

The water hammer conditions are

$$
\begin{aligned}
H(X, \tau=0) & =1-X, V(X, \tau=0)=1 \\
H(X=0, \tau>0) & =1, V(X=1, \tau>0)=0 .
\end{aligned}
$$

An explanation of the dimensionless parameters, the $C_{i}, i=1, \ldots, 5$, also appears in [10] and [14, and is briefly restated here for completeness

- $C_{1}$ : Advection Effect. $C_{1}$ may be written as $\left(\rho v_{\infty}^{2}\right) /\left(\rho g h_{12}\right)$ and then is seen as the ratio of a velocity head to a hydraulic head.

- $C_{2}$ : Viscous Effect. $C_{2}$ is the kinematic 'viscous head' relative to the applied hydraulic head $h_{12}$ difference.

- $C_{3}$ : Inertial Effect. This ratio of an inertial head to applied hydraulic head difference is more relevant as turbulence increases.

- $C_{1} C_{4}$ : Advective Head. The product $C_{1} C_{4}$ may be recast in the form $\left[\rho g h_{12} /\left(\rho c_{p}^{2}\right)\right]$, whereupon it is the ratio of hydraulic head difference (expressed as a pressure) to the pressure from the propagating wave.

- $C_{4}$ : Local Velocity. $\sqrt{C_{4}}=\left(\rho g h_{12}\right) /\left(\rho c_{p} v_{\infty}\right)$ is the applied hydraulic head difference relative to the 'local' pressure head.

- $C_{5}$ : Elevation Head. This is elevation head relative to propagating wave celerity head.

In the applications considered here, $C_{1}, C_{2}$ and $C_{3}$ are $O(1)$ ('O' means 'the order of') while the wave-speed celerity $O\left(c_{p}\right) \gg 1$ so that $C_{4}$ and $C_{5}$ are small. If $\epsilon=O\left(1 / \sqrt{c_{p}}\right)$ then $C_{1} C_{4}=C_{1} c_{4} \epsilon^{2}, C_{4}=c_{4} \epsilon^{2}$, and $C_{5}=c_{5} \epsilon^{2}$ with $C_{1}, c_{4}$ and $c_{5}$ treated as order one. 
Using these definitions,

$$
\begin{array}{r}
H_{X}+C_{1} V V_{X}+V_{\tau}+C_{2} V+C_{3} V|V|+B_{1} V_{\tau}+B_{1} B_{2} \operatorname{sign}(V)\left|V_{X}\right|=0 \\
V_{X}+C_{1} c_{4} \epsilon^{2} V H_{X}+c_{4} \epsilon^{2} H_{\tau}+c_{5} \epsilon^{2} V=0 .
\end{array}
$$

The boundary and initial conditions are unaffected because they do not contain any dimensionless parameters.

5. Water hammer time scales. The water hammer is characterized in terms of two time-scales: (i) a slowly evolving trend (rigid-column motion) operating at a time-scale $\tau$, and (ii) a superimposed wave whose amplitude attenuates over the long time-scale $\tau$ and has a period of oscillation at the short time-scale $t=\tau / \epsilon$. The reduction in velocity at the downstream end $X=L$, at time $t=0$ from $V=1$ to $V=0$, initiates a backward travelling pressure-wave. The head is proportional to $\sqrt{c_{p}}=1 / \epsilon$ and, as $\epsilon \rightarrow 0$, the wave celerity approaches infinity and the head necessarily becomes singular. The asymptotic expansion of the head is regularized with respect to $\epsilon \rightarrow 0$ by setting $\tilde{H}=\epsilon H$.

The multiple-scales analysis is simplified by noting that the water hammer equations are invariant with respect to flow reversals. Specifically, this point can be observed under the substitution $Y=1-X$ and $W=-V$ [14], and we find that the invariance remains true in the presence of unsteady friction.

Therefore, working in terms of $\tau$ and $t$, defining a regularized head $\tilde{H}$, and noting the invariance of the momentum/continuity equations to flow direction, the multiple-scales form of (8) and (9) is

$$
\begin{array}{r}
\tilde{H}_{X}+\epsilon C_{1} V V_{X}+\epsilon V_{\tau}+V_{t}+\epsilon C_{2} V+\epsilon C_{3} V^{2}+\epsilon B_{1} V_{\tau}+B_{1} V_{t}-\epsilon B_{1} B_{2} V V_{X}=0 \\
V_{X}+C_{1} c_{4} \epsilon V \tilde{H}_{X}+c_{4} \epsilon \tilde{H}_{\tau}+c_{4} \tilde{H}_{t}+c_{5} \epsilon^{2} V=0
\end{array}
$$

with conditions

$$
\begin{gathered}
\tilde{H}(X, \tau=0)=\epsilon(1-X), V(X, \tau=0)=1 \\
\tilde{H}(X=0, \tau>0)=\epsilon, V(X=1, \tau>0)=0 .
\end{gathered}
$$

Note that conditions satisfied here by the pressure-wave attenuation will be independent of the flow direction, and thus reference to the invariant form in (10) and (11) will be sufficient; however, more general conditions may violate this required invariance.

6. Multiple-scales expansion. Each of the head and velocity are written as the superposition of an attenuating wave and a trend. The wave component [10] is written in a generalized form required for the inclusion of unsteady friction. The attenuating wave component for the head and velocity respectively is a series with terms of the form $P_{j}(\tau) F_{n j}\left(\alpha_{n}(X-c t)\right)$ and $P_{j}(\tau) G_{n j}\left(\alpha_{n}(X-c t)\right)$. The $P_{j}(\tau)$ are the long time-scale pressure-wave attenuation functions while $F_{n j}\left(\beta_{n}(X-c t)\right)$ and $G_{n j}\left(\beta_{n}(X-c t)\right)$ are the oscillatory wave components with dimensionless wave speed $c=1 / \sqrt{\left(1+B_{1}\right) c_{4}}$. The $\alpha_{n}$ and $\beta_{n}$ are complex modes with imaginary part $n \mathbf{i}$. The subscript $j$ corresponds to the order of expansion $\epsilon^{j}$. The trending component [14] for the regularized head 
is $\tilde{H}(X, \tau)=\epsilon H_{0}(X)+\epsilon^{2} H_{1}(X, \tau)+\ldots$ while the velocity is $V_{0}(\tau)+\epsilon V_{1}(X, \tau)+\ldots$. Altogether these yield the multiple-scales expansion

$$
\begin{aligned}
\tilde{H}(X, t, \tau)= & \sqrt{\frac{1+B_{1}}{c_{4}}} P_{1}(\tau)\left(1+\epsilon Q_{11}(\tau)+\ldots\right)\left[F_{10}\left(\alpha_{1}(X-c t)\right)+\epsilon F_{11}\left(\alpha_{1}(X-c t)\right)+\ldots\right] \\
& +\sqrt{\frac{1+B_{1}}{c_{4}}} P_{2}(\tau)\left(1+\epsilon Q_{21}(\tau)+\ldots\right)\left[F_{20}\left(\alpha_{2}(X-c t)\right)+\epsilon F_{21}\left(\alpha_{2}(X-c t)\right)+\ldots\right] \\
& +\ldots \\
& +\epsilon H_{0}(X)+\epsilon^{2} H_{1}(X, \tau)+\ldots \\
V(X, t, \tau)= & P_{1}(\tau)\left(1+\epsilon \tilde{Q}_{11}(\tau)+\ldots\right)\left[G_{10}\left(\beta_{1}(X-c t)\right)+\epsilon G_{11}\left(\beta_{1}(X-c t)\right)+\ldots\right] \\
& +P_{2}(\tau)\left(1+\epsilon \tilde{Q}_{21}(\tau)+\ldots\right)\left[G_{20}\left(\beta_{2}(X-c t)\right)+\epsilon G_{21}\left(\beta_{2}(X-c t)\right)+\ldots\right] \\
& +\ldots \\
& V_{0}(\tau)+\epsilon V_{1}(X, \tau)+\ldots
\end{aligned}
$$

The first approximation to the trending head and velocity which depends upon $X$ and $\tau$ is found by substituting (13) to (10) and (11), and this yields

$$
\begin{array}{r}
H_{0}(X)=1 \\
V_{0}(\tau)=\frac{\tilde{D} e^{-C_{2} \tau}}{1-\tilde{D} C_{3} / C_{2} e^{-C_{2} \tau}}
\end{array}
$$

where the constant $\tilde{D}$ is determined later. In the following section, the wave attenuation function associated with the unsteady friction model is found using (13).

7. Attenuation function. The first-mode attenuation function $P_{1}(\tau)$ is found here, and the numerics will show that this is the dominant mode of the wave attenuation.

Starting with $\epsilon^{0}$ and substituting (13) to each of (10) and (11) we see that the zeroth approximation to the head and velocity of (13) must satisfy $\tilde{H}_{X}+\left(1+B_{1}\right) V_{t}=0$ and $V_{X}+c_{4} \tilde{H}_{t}=0$.

Continuing to first order in $\epsilon$ with the momentum equation (10) and continuity equation (11), we find respectively that

$$
\begin{array}{r}
P_{1 \tau}(\tau)+\left[\frac{\alpha_{1} C_{1} V_{0}(\tau)+2 C_{3} V_{0}(\tau)-\alpha_{1} B_{1} B_{2}}{1+B_{1}}\right] P_{1}(\tau)+\frac{1}{\sqrt{c_{4}\left(1+B_{1}\right)}} \alpha_{1} P_{1}(\tau) \Delta Q_{11}=0 \\
\frac{1}{\sqrt{c_{4}\left(1+B_{1}\right)}} \alpha_{1} P_{1}(\tau) \Delta Q_{11}=\alpha_{1} C_{1} V_{0}(\tau)+P_{1 \tau}(\tau)
\end{array}
$$

where $\Delta Q_{11}=Q_{11}-\tilde{Q}_{11}$ and $V_{0}(\tau)$ is in (14). Substituting (16) to (16) we eliminate $\Delta Q_{11}$, and the first-mode attenuation satisfies

$$
P_{1 \tau}(\tau)+\lambda(\tau) P_{1}(\tau)=0
$$

where the complex rate is

$$
\lambda(\tau)=\left[\frac{\left(\alpha_{1} C_{1}\left(2+B_{1}\right)+2 C_{3}\right) V_{0}(\tau)+C_{2}-\alpha_{1} B_{1} B_{2}}{2\left(1+B_{1}\right)}\right] .
$$


The magnitude of the first-mode attenuation $P_{1}(\tau)$ follows as

$$
\left|P_{1}(\tau)\right|=\tilde{A} e^{-\int_{0}^{\tau} \Re(\lambda(z)) d z}
$$

where $\tilde{A}=\left|P_{1}(0)\right|$ is a free constant.

The two free constants $\tilde{A}$ and $\tilde{D}$ are found similarly to the approach used in [14] while the real part of the third constant $\alpha_{1}$ is found numerically.

Firstly, the initial condition $P_{1}(0)=1$ ensures that the head does not exceed $1 / \sqrt{c_{4}}$ at $\tau=0$ and this gives $\tilde{A}=1 / P(0)$. Secondly, the constant $\tilde{D}$ would generally require a numerical optimization involving a comparison of the numerical and analytic approximations to the attenuation function to obtain an optimal value of $D$, e.g. via least squares. However, a nearly optimal choice of $\tilde{D}$ may be found by setting $V(0)=1 / 2$. This choice implies the first-order trend $V_{0}(\tau)$ approximates the periodic average of $V(X, \tau)$.

Given $\tilde{A}$ and $\tilde{D}$ we find, after approximating for $\left(B_{1}, C_{1}\right) \ll 1$ viz. Table 1, a somewhat simplified form of the pressure-wave attenuation

$$
\left|P_{1}(\tau)\right|=e^{\Re\left(\alpha_{1}\right) B_{1} B_{2} \tau / 2} \frac{e^{-C_{2} \tau / 2}}{1+C_{3} /\left(2 C_{2}\right)\left(1-e^{-C_{2} \tau}\right)},
$$

and if the viscous head linear term in the extended steady friction model $C_{2} V+C_{3} V^{2}$ is zeroed, i.e. $C_{2}=0$,

$$
\left|P_{1}(\tau)\right|=e^{\Re\left(\alpha_{1}\right) B_{1} B_{2} \tau / 2} \frac{1}{1+C_{3} \tau / 2} .
$$

Hence, the contribution made by unsteady friction in comparison to the steady friction model appears as the factor $\exp \left(\Re\left(\alpha_{1}\right) B_{1} B_{2} \tau / 2\right)$. The unknown constant $\Re\left(\alpha_{1}\right)$ is determined in the following section.

8. Results. The pressure-wave attenuation has a weak spatial dependence since its first approximation only depends upon $\tau$, and therefore it approximates the periodicaverage of the pressure-wave during a pseudo-period (the water hammer traverses the pipe four times [13] during a pseudo-period). During a single period, the unsteady friction shuttles between a nearly zero value when the flow kinetic energy is decreasing (travelling upstream in our application) and a maximal value when the flow kinetic energy is increasing (travelling downstream in our application). A nearly optimal choice of $\Re\left(\alpha_{1}\right)=-1$ yields $\left|P_{1}(\tau)\right|$ that approximates the periodic-average of the pressure-wave during a pseudo-period.

All results are presented in Figure 1, and their derivation is described within the caption. The wave attenuation is numerically computed as the absolute difference $\mid \tilde{H}(X, \tau)-$ $\tilde{H}(X-2 \Delta X, \tau) \mid$ of the numerical solution. It is found at three dimensionless positions: $X=0.1$ (near upstream entrance), $X=0.5$ (midway along the pipe) and $X=0.9$ (near the downstream end). The same symbols are used for results at each position to enable a comparison between the steady and unsteady friction models. In all cases, the first-mode attenuation function $\left|P_{1}(\tau)\right|$ is compared to the numerical solution for physical parameters given in Table 1 that were taken from a physical study [1] (more details of the apparatus used in [1] are available in [3]). The Brunone coefficient $k$ in Table 1 was estimated [1] from the Vardy-Brown decay shear coefficient [8] and a low Reynolds number turbulent flow. 


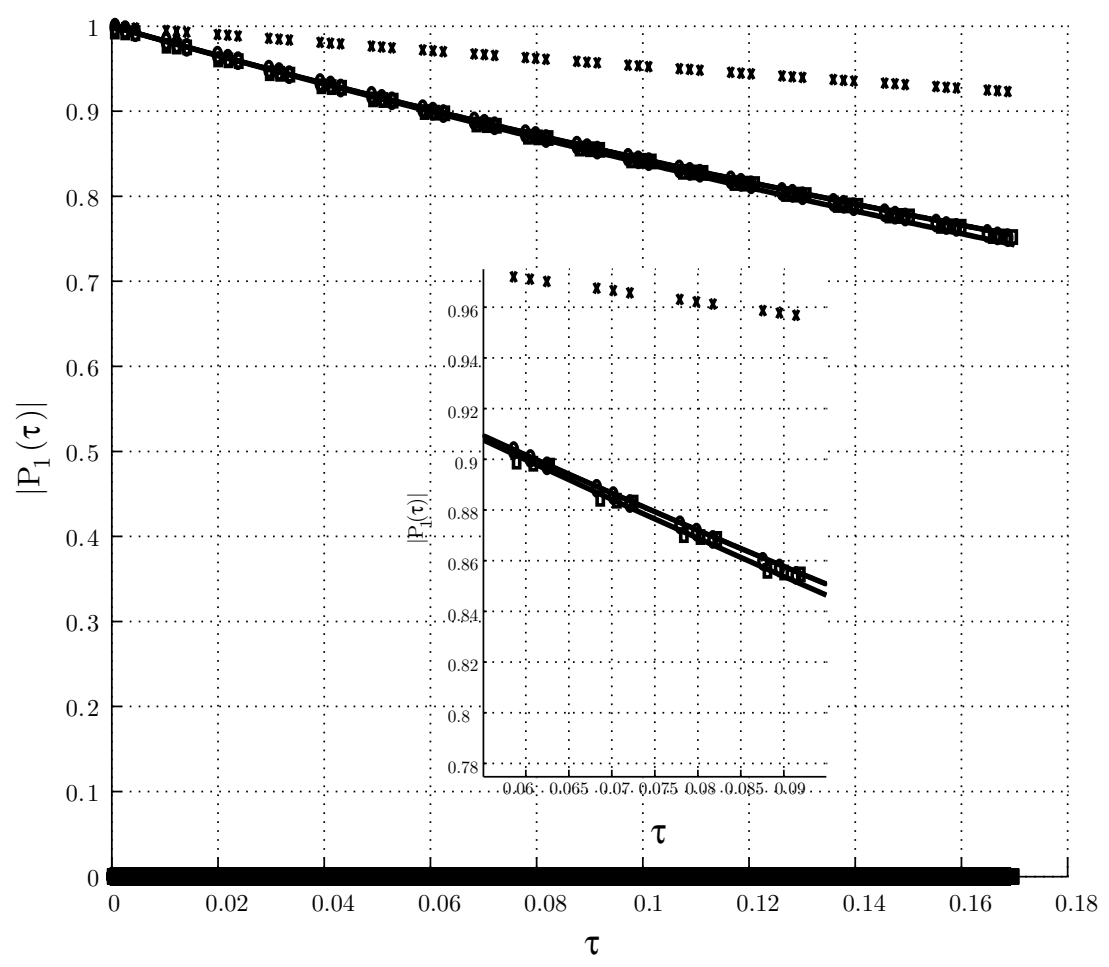

FIG. 1. The numerically determined pressure-wave attenuation is shown for three cases: (i) the steady friction model (crosses) with zero viscous head $\left(C_{2}=0\right)$ and zero unsteady friction $\left(B_{1}=0, B_{2}=\right.$ 0 ) in (4), (ii) the Brunone unsteady friction model (squares) with zero viscous head $\left(C_{2}=0\right)$ and unsteady parameters $B_{1}=0.0123$, $B_{2}=207$ from [1] (see Table 1), and (iii) the extended steady friction model (circles) with non-zero viscous head $C_{2}=B_{1} B_{2}$ and zero unsteady friction terms. The wave attenuation is computed as the absolute difference $|\tilde{H}(X, \tau)-\tilde{H}(X-2 \Delta X, \tau)|$ of the numerical solution at dimensionless positions $X=0.1$ (near upstream entrance), $X=0.5$ (midway along the pipe) and $X=0.9$ (near the downstream end). These three locations are not distinguished in the results presented in each of (i), (ii) and (iii) above. Note that absolute differences of head that remain relatively constant in time appear as the heavier line of smaller values along the bottom of the figure while rapid changes in head at each $X$ location are the remaining values that approximate the wave amplitude. The analytical pressure-wave attenuation closely approximates the numerical results in case (ii) as the solid line and in case (iii) as the dashed line.

Firstly, the attenuation found numerically for the unsteady friction model, and the steady friction model with and without the viscous head term $C_{2} V$ is presented in the figure. The numerical results for the steady friction model attenuation are found without viscous head $\left(C_{2}=0\right)$ and without unsteady friction $\left(B_{1}=0, B_{2}=0\right)$, and they appear near the top (crosses). The numerical results for attenuation based on unsteady friction parameters $B_{1}=0.0123, B_{2}=207$ and zero viscous head $\left(C_{2}=0\right)$ are shown as squares. 


\begin{tabular}{|c|}
\hline Dimensional parameters \\
\hline Conduit diameter $D_{c}=0.0221 \mathrm{~m}$ \\
\hline Conduit length $L=37.23 \mathrm{~m}$ \\
\hline Conduit roughness $\epsilon_{c}=0.0003 \mathrm{~m}$ \\
\hline Conduit wall thickness $w=0.0016 \mathrm{~m}$ \\
\hline Applied head change at $t=0, h_{12}=h_{1}-h_{2}=0.13 \mathrm{~m}$ \\
\hline Young's modulus of iron $E=2.0 \cdot 10^{11} \mathrm{~Pa}$ \\
\hline Water bulk modulus $E_{v}=2.27 \cdot 10^{9} \mathrm{~Pa}$ \\
\hline Kinematic viscosity $\nu=\mu / \rho=10^{-6} \mathrm{~m}^{2} / \mathrm{s}$ \\
\hline Brunone coefficient $k=0.0245$ \\
\hline Computed dimensional parameters \\
\hline Steady velocity $v_{\infty}=0.2 \mathrm{~m} / \mathrm{s}$ \\
\hline Celerity $c_{p}=1319 \mathrm{~m} / \mathrm{s}$ \\
\hline Inertial constant $s=3.25 \mathrm{~s}^{2} / \mathrm{m}$ \\
\hline Dimensionless parameters \\
\hline $\begin{array}{c}\left.C_{1}=0.0318, C_{2}=0, C_{3}=1 \text { (Momentum equation (8) }\right) \\
C_{1} c_{4}=7.15 \cdot 10^{-4}, c_{4}=0.0228, c_{5}=-7.33 \cdot 10^{-4}, \epsilon^{2}=10^{-3} \text { (Continuity equation (9)) } \\
\left.B_{1}=0.0123, B_{2}=207 \text { (Brunone unsteady friction (8) }\right)\end{array}$ \\
\hline TABLE 1. The set of parameters used for the valve-closure water \\
\hline hammer. Dimensional, computed dimensional, and dimensionless \\
\hline parameters are separated for clarity. These parameters are taken \\
\hline $\begin{array}{l}\text { from the physical model presented in }[1] \text { and studied further in }[2] \text {. } \\
\text { Note that the Brunone coefficient } k \text { is estimated in } 1 \text { from Vardy- }\end{array}$ \\
\hline $\begin{array}{l}\text { Brown's shear decay coefficient }[8, \text { and viscous constant } r \text { is de- } \\
\text { scribed within the figure caption and the results section in } 8\end{array}$ \\
\hline
\end{tabular}

In both the steady and unsteady friction models the inertial head coefficient $C_{3}=1$. The increased pressure-wave attenuation predicted by the Brunone model of unsteady friction over the steady friction model is apparent.

Secondly, the analytically computed wave attenuation of equation (21) (solid line) for the unsteady friction model with $C_{2}=0, B_{1}=0.0123$ and $B_{2}=207$ closely approximates the attenuation found numerically (squares). The analytical wave attenuation in equations (20) and (21) shows that the increased attenuation due to unsteady friction for weak spatial dependence where the first approximation to the attenuation depends only on $\tau$ may be reduced to the factor $\exp \left(\Re\left(\alpha_{1}\right) B_{1} B_{2} \tau / 2\right)$.

An interesting possibility, raised by the analytical form of the wave attenuation in (20) and (21), is the possibility of a viscous basis for the increased wave attenuation seen in the unsteady friction results (squares). The form of (20) and (210) indicate that the Brunone unsteady friction results (squares) may be approximated via the steady model if the viscous head $C_{2} V$ is included as $C_{2}=B_{1} B_{2}$, followed by zeroing unsteady friction terms. The attenuation $\left|P_{1}(\tau)\right|$ (21) with $C_{2}=B_{1} B_{2}$ is shown in Figure 1, with details in the inset, and it closely approximates the unsteady model wave attenuation. The numerical computations show that the dynamics of the unsteady friction model (squares) are also closely matched by the extended steady friction model (circles) to within a phase-shift of $\tau \approx 0.0004$.

The link observed here between the extended steady friction and unsteady friction models is possible when the pressure-wave attenuation has a weak spatial dependence 
and is only dependent upon $\tau$ to a first approximation. For example, in the presence of cavitation ([6] and [7]) or a valve closed at a time-scale approaching the water hammer transit time (4]), such a link would not exist due to increased spatial dependence of the pressure-wave attenuation.

9. Conclusions. A multiple-scales asymptotic analysis was developed for pressurewave attenuation in the Brunone model of unsteady friction. The analytical form showed that the increased attenuation due to unsteady friction over the standard steady friction model may be reduced to a single time-dependent exponential factor dependent on the product of the Brunone unsteady friction parameters. The method was applied to water hammer caused by sudden valve closure in water reservoir pipelines in [1]. The analytical form of the pressure wave attenuation also predicted that viscous head in an extended steady friction model may account for increased pressure-wave attenuation seen in the unsteady friction model when the attenuation has a weak spatial dependence. The approach used here should be useful to explain the physical basis of pressure-wave attenuation predicted in other water hammer models.

\section{REFERENCES}

[1] A. Bergant, A. R. Simpson, and J. Vitkovsky, Review of unsteady friction models in transient pipe flow, The Work Group On The Behaviour Of Hydraulic Machinery Under Steady Oscillatory Conditions, 1999, 12pp.

[2] A. Bergant and A. S. Tijsseling, Parameters affecting water-hammer wave attenuation, shape and timing, Proceedings of the 10th International Meeting of the IAHR Work Group on the Behaviour of Hydraulic Machinery under Steady Oscillatory Conditions, 2001, Paper C2, 12pp.

[3] A. Bergant, A. S. Tijsseling, J. P. Vitkovsky, D. I. C. Covas, A. R. Simpson, and M. F. Lambert, Parameters affecting water-hammer wave attenuation, shape, and timing part ii: Case studies, IAHR J. of Hydraulic Research 46(3) (2008), 382-391.

[4] A. Bergant, J. Vitkovsky, A. R. Simpson, and M. Lambert, Performance of instantaneous acceleration models of unsteady skin friction in practical applications, 3rd Unsteady Friction Group Meeting, University of Dundee, 2002, 14pp.

[5] B. Brunone and U. M. Golia, Improvements in modelling of water hammer and cavitating flow - experimental verification, Proc. of the XXII Italian Conference on Hydraulics and Hydraulic Constructions, 1990, pp. 147-160.

[6] - Some considerations on velocity profiles in unsteady pipe flows, Proc. of the Int. Conf. on Entropy and Energy Dissipation in Water Resources, 1991, pp. 481-487.

[7] B. Brunone, U. M. Golia, and M. Greco, Some remarks on the momentum equations for fast transients, Hydraulic Transients with Column Separation (9th and Last Round Table of the IAHR Group), IAHR, 1995, pp. 201-209.

[8] Vardy A. E. and Brown J. M. B., Transient, turbulent, smooth pipe flow, Proc. Int. Conf. on Pressure Surges and Fluid Transients, BHR Group, 1996, pp. 289-311.

[9] M. Greco, Recent findings on column separation during water hammer, G.N.I. Edizioni Libreria Progetto, 1990, pp. 261-272.

[10] S. Y. Han, D. Hansen, and G. Kember, Multiple scales analysis of water hammer attenuation, Quart. Appl. Math. 69 (2011), no. 4, 677-690, DOI 10.1090/S0033-569X-2011-01258-9. MR2893995 (2012j:76072)

[11] Golia U. M., On the evaluation of the friction term in water hammer, Dept. of Hydraulics, Univ. of Napoli 'Frederico II', 1990, Rep. 639.

[12] J. P. Vitkovsky and A. R. Simpson, A critique of the Brunone et al. unsteady state friction method, Tech. report, Hydraulics Technical Memorandum No. 90/1, Dept. of Civil and Envir. Engrg., University of Adelaide, 1998. 
[13] E. B. Wylie and V. L. Streeter, Fluid transients in systems, Prentice-Hall, Englewood Cliffs, NJ, 1993.

[14] E. Yao, G. C. Kember, and D. H. Hansen, Analysis of water hammer attenuation in sudden valve closure applications, J. Engng. Mech. (submitted). 